\title{
Characteristics of Coulomb fission
}

\author{
Volker Oberacker and Walter Greiner* \\ Physics Division, Oak Ridge National Laboratory, Oak Ridge, Tennessee 37830
}

Hans Kruse and William T. Pinkston

Department of Physics, Vanderbilt University, Nashville, Tennessee 37235

(Received 23 February 1979)

\begin{abstract}
Within an extended semiquantal theory we perform large-sized coupled-channel calculations involving 260 collective levels for Coulomb fission of ${ }^{238} \mathrm{U}$. Differential Coulomb fission cross sections are studied as a function of bombarding energy and impact parameter for several projectiles. In the $\mathrm{Xe}+\mathrm{U}$ case, total cross sections are also given. We find a strong dependence on projectile charge number, $P^{\mathrm{CF}}\left(180^{\circ}\right) \sim\left(Z_{p}\right)^{6}$ in the region $50 \leq Z_{p} \leq 92$ for a fixed ratio $E / E_{\text {Coul }}$, which might be helpful to separate Coulomb fission experimentally from sequential fission following transfer reactions. Since the cross sections are sensitive to the moment of inertia $\Theta$ at the saddle point, Coulomb fission can serve as a tool to investigate the dependence of $\Theta$ on elongation. The fragment angular distribution exhibits deviations from $1 / \sin \theta_{f}$ which are pronounced at low incident energies. Our theory indicates that the recently measured $\mathrm{Xe}+\mathrm{U}$ fission cross sections contain a major fraction of Coulomb-induced fission at $E \leq 0.85 E_{\text {Coul. }}$.
\end{abstract}

NUCLEAR REACTIONS, FISSION Calculated Coulomb fission cross sections $\sigma\left(E_{p}, \theta_{p}\right)$ for ${ }_{54} \mathrm{Xe},{ }_{67} \mathrm{Ho},{ }_{82} \mathrm{~Pb},{ }_{92} \mathrm{U} \rightarrow{ }_{92}^{238} \mathrm{U}$, fragment angular distribution, fission energy spectrum, mean spin value $\left\langle J_{\boldsymbol{f}}\right\rangle$.

\section{INTRODUCTION}

Coulomb fission, i.e., fission of a deformed target nucleus induced by the time-varying Coulomb field of a heavy projectile, was first studied for adiabatic collisions in 1966 by Guth and Wilets ${ }^{1}$ within a classical model. Their main motivation to study this process was that Coulomb.interaction directly couples to the collective fission degree of freedom in contrast to other fission mechanisms presently known which proceed indirectly via compound nucleus formation with a typical lifetime $T_{f} \approx 10^{-15}-10^{-16} \mathrm{~s}$. Consequently, Coulomb fission (CF) should develop much faster and is expected to be a suitable probe to investigate collective potential energy surfaces at high excitation energy and large deformations.

During the last years, several classical and semiquantal CF models ${ }^{2-6}$ have been worked out. Even though the theories all predict Coulomb fission for actinide target nuclei (low fission barrier) and projectiles with charge numbers $Z_{P} \gtrsim 50$, the calculated cross sections differ by about 3 orders of magnitude. Early attempts to measure this new process with medium-mass nuclei failed, ${ }^{7,8}$ but recently fission events induced by Xe ions below $E_{\text {Coul }}$ have been detected at the heavy-ion accelerators in Berkeley ${ }^{9}{ }^{10}$ and Darmstadt GSI. In particular, the counter experiments ${ }^{11}$ and radiochemical experiments ${ }^{12}$ performed at GSI show some indication for Coulomb fission at low bombarding energies. A unique experimental proof, however, requiring the identification of the backscattered projectilelike nucleus has not been carried out so far. In previous work ${ }^{5,6}$ we have shown that nuclear structure effects like rotation-vibration interaction (RVI) may considerably influence the CF cross sections. The main reason is that the neglect of RVI results in a preference of fission from high-spin members of the ground-state rotational band, ${ }^{4}$ which overestimates the experimental limits by at least 1 order of magnitude. At low bombarding energies, a fair agreement between our theory ${ }^{5}$ and the data of Habs et al. ${ }^{11}$ was found. Nevertheless, several open questions remain concerning the details of the process, namely: (a) Are there any characteristic features which might help to separate CF from other competing fission processes like sequential fission following transfer or deep-inelastic reactions? (b) How important are collective rotations in the $C F$ process? Up to now, only extreme cases have been studied. Of particular interest in this connection is the strong sensitivity of CF on the dependence of the moment of inertia on deformation. It seems that we can learn from CF the behavior of $\Theta\left(a_{0}\right)$ at large elongations, especially by using high- $Z$ projectiles. (c) What is the time scale involved in Coulomb fission? In the limit of an infinitely slow. collision, ${ }^{1}$ an actinide nucleus is expected to fission near the distance of closest approach where the. barrier of the effective fission potential $V_{\text {eff }}=V_{\text {target }}$ $+V_{\text {Coul }}$ vanishes ("prompt" CF). In this case, the fragments emerge preferentially at angles $\theta_{f} \sim 90^{\circ}$ to the beam axis. As first pointed out by Wilets, Guth, and Tenn, ${ }^{2}$ the collision is not adiabatic.

1453 (c) 1979 The American Physical Society 
According to their dynamical classical calculations for $\mathrm{Xe}+\mathrm{U}$, fission occurs at about $90 \mathrm{fm}$ on the outgoing branch of the trajectory (see Fig. 6 of Ref. 2). The fragment angular distribution, however, peaks at $90^{\circ}$ as expected for a fast fission process. (d) Does damping into noncollective configurations play a significant role?

In this article we will concentrate on questions (a) and (b). Distinct from a classical description, where the nucleus fissions instantaneously, our semiquantal formulation allows to evaluate the probability to find prompt fission near the turning point $r_{\text {min }}$. According to Ref. 13, this fast component is found to be negligible even in $\mathrm{U}+\mathrm{U}$ collisions. The damping question is less important in Coulomb fission (see Sec. II) and will be dealt with in a forthcoming detailed theoretical article. ${ }^{14}$ The paper is organized as follows: In Sec. II we give a brief description of our semiquantal CF formalism which treats the continuum fission problem exactly by means of a projection operator technique. The final computations, of course, involve some approximations. In Sec. III we specify the nuclear model and discuss the results for Coulomb fission of ${ }^{238} \mathrm{U}$ induced by various projectiles $\left(54 \leqslant Z_{p} \leqslant 92\right)$. In particular, we investigate $\sigma^{\mathrm{CF}}\left(E_{p}, \theta_{p}\right)$ and the fragment angular distribution at $\theta_{p}^{\text {c.m. }}=180^{\circ}$. The theoretical predictions for the $\mathrm{Xe}+\mathrm{U}$ system are compared with recent experimental data. Section IV summarizes the results and gives a short outlook.

\section{OUTLINE OF THE THEORY}

In this section the semiquantal theory is outlined with the principal results presented. The theory will be presented in more detail in a later paper. In the semiquantal theory, the relative motion of the colliding nuclei is treated classically. This is possible in heavy-ion scattering because of the large value of the Sommerfeld parameter, $\eta$ $=Z_{1} Z_{2} e^{2} / \hbar v$. The excitation of internal states is treated quantum mechanically; it is the result of the time-dependent monopole-quadrupole parts of the electromagnetic and strong interactions between the colliding nuclei. Monopole-monopole contributions are assumed to affect only the relative motion; higher multipole-multipole contributions are known ${ }^{15}$ to be almost negligible. The internal dynamics of either nucleus is governed by a Schrödinger equation of the form

$$
\left(H\left(\xi_{i}\right)+V\left(\xi_{i}, \overrightarrow{\mathrm{r}}(t)\right)-i \hbar \frac{\partial}{\partial t}\right) \psi\left(\xi_{i}, t\right)=0 .
$$

The symbols $\xi_{i}$ and $H$ represent the intrinsic coordinates and Hamiltonian of the nucleus under consideration, and $\overrightarrow{\mathrm{r}}(t)$ is the relative distance vector, taken in lowest order to correspond to a Rutherford trajectory. The interaction $V$ is the monopolequadrupole interaction.

Except for simple one-dimensional cases, a direct numerical integration of Eq. (2.1) is impractical. We therefore expand the wave function, $\psi\left(\xi_{i}, t\right)$, into an appropriate basis. In choosing the basis, one must take into account three different kinds of states: (i) collective bound states, (ii) collective continuum states (final states of the fission process), and (iii) single-particle type excitations. The collective bound states are strongly excited by the Coulomb excitation process, whereas the single-particle states are weakly excited. We will see later that the main importance of the noncollective states is their coupling to excited collective states. This results in a damping, i.e., reduction of the fission probability. In what follows we neglect single-particle states and treat only the collective degrees of freedom.

Since the actinides spontaneously fission, there are no states which are truly bound against decay into states of type (ii). The long-lived quasibound states of these nuclei are continuum resonances. They can be treated as bound states embedded in a continuum by projection techniques, such as those of Feshbach, ${ }^{16}$ Wang and Shakin, ${ }^{17}$ and Micklinghoff. $^{18}$ It is possible to find a set of bound (i.e., normr!izable) states $\left|\phi_{\mu}\right\rangle$ and projectors $P_{\mu}$ such that : can be decomposed as follows:

$$
l_{c}=\sum_{\mu} P_{\mu} H_{c} P_{\mu}+Q H_{c} Q+\sum_{\mu}\left(P_{\mu} H_{c} Q+Q H_{c} P_{\mu}\right) \text {. }
$$

The operator $Q$ is given by

$$
Q=1-\sum_{\mu} P_{\mu} \text {. }
$$

(We have written $H$ as $H_{c}$ to emphasize that we treat only collective degrees of freedom.) The eigenstates of $Q$ are continuum states, orthogonal to the $\left|\phi_{\mu}\right\rangle$. If the $\left|\phi_{\mu}\right\rangle$ are properly chosen, the continuum states will all be nonresonant. For the basis used in expanding $\psi$, we choose eigenstates of the zero-order Hamiltonian

$$
H_{0}=\sum_{\mu} P_{\mu} H_{c} P_{\mu}+Q H_{c} Q \text {. }
$$

These states have the properties

$$
\begin{aligned}
& H_{0}\left|\phi_{\mu}\right\rangle=\epsilon_{\mu}\left|\phi_{\mu}\right\rangle, \quad H_{0}\left|\phi_{E}\right\rangle=E\left|\phi_{E}\right\rangle, \\
& \left\langle\phi_{\mu} \mid \phi_{\nu}\right\rangle=\delta_{\mu \nu}, \quad\left\langle\phi_{E} \mid \phi_{E}\right\rangle=\delta\left(E-E^{\prime}\right), \\
& \left\langle\phi_{\nu} \mid \phi_{E}\right\rangle=0 .
\end{aligned}
$$

The expansion of $\psi\left(\xi_{i}, t\right)$ of Eq. (2.1) is given by

$$
\begin{aligned}
\psi\left(\xi_{i}, t\right)= & \sum_{\mu} a_{\mu}(t)\left|\phi_{\mu}\right\rangle e^{-i \epsilon_{\mu} t / \hbar} \\
& +\int d E b_{E}(t)\left|\phi_{E}\right\rangle e^{-i E t / \hbar} .
\end{aligned}
$$


If Eq. (2.5) is substituted into Eq. (2.1), a set of coupled equations for the $a_{\mu}$ and $b_{E}$ amplitudes results:

$$
\begin{aligned}
& i \hbar \dot{a}_{\mu}(t)=\sum_{\nu}\left\langle\phi_{\mu}|V(t)| \phi_{\nu}\right\rangle a_{\nu}(t) \exp \left(\frac{i}{\hbar}\left(\epsilon_{\mu}-\epsilon_{\nu}\right) t\right)+\int d E b_{E}(t)\left\langle\phi_{\mu}\left|\left[H_{c}+V(t)\right]\right| \phi_{E}\right\rangle \exp \left(\frac{i}{\hbar}\left(\epsilon_{\mu}-E\right) t\right) \\
& i \hbar \dot{b}_{E}(t)=\sum_{\nu}\left\langle\phi_{E}\left|\left[H_{c}+V(t)\right]\right| \phi_{\nu}\right\rangle a_{\nu}(t) \exp \left(\frac{i}{\hbar}\left(E-\epsilon_{v}\right) t\right)+\int d E^{\prime} b_{E}(t)\left\langle\phi_{E}|V(t)| \phi_{E^{\prime}}\right\rangle \exp \left(\frac{i}{\hbar}\left(E-E^{\prime}\right) t\right) .
\end{aligned}
$$

We assume it is a good approximation to neglect the continuum rearrangement terms, i.e., those depending on $\left\langle\phi_{E}|V| \phi_{E},\right\rangle$. A simple physical interpretation can be applied to each of the remaining terms. The matrix elements $\left\langle\phi_{\mu}|V| \phi_{\nu}\right\rangle$ account for the Coulomb excitation of the bound states. Terms involving $\left\langle\phi_{\nu}\left|H_{c}\right| \phi_{E}\right\rangle$ result in spontaneous decay of bound states into the continuum; $\left\langle\phi_{\nu}|V| \phi_{E}\right\rangle$ terms result in emission stimulated by the Coulomb field. This stimulated emission is strongly time dependent and takes place near the distance of closest approach. It corresponds to what has been called "prompt fission" and can be thought of physically as the escape of the fission fragments as a result of the lowering of the fission barrier by the perturbation $V\left(\xi_{2}, \overrightarrow{\mathbf{r}}(t)\right)$. Studies of direct numerical integration of Eq. (2.1), based on a simple one-dimensional model, ${ }^{13}$ indicate that prompt fission events can be neglected. We, therefore, neglect those terms in Eqs. (2.6a) and (2.6b) which involve $\left\langle\phi_{\nu}|V| \phi_{E}\right\rangle$.

Even further restrictions must be placed on the system to make its solution tractable. In this paper we make the assumption of "asymptotic Coulomb fission," 5 i.e., that the process proceeds in two steps. The first step is the Coulomb excitation of the bound states. The second step is the radioactive decay of these states. The dynamics of the first step are described by the coupled system

$$
\begin{array}{r}
i \hbar \stackrel{\circ}{\nu}_{\nu}(t) \simeq \sum_{\mu} a_{\mu}(t)\left\langle\phi_{\nu}|V(t)| \phi_{\mu}\right\rangle \\
\times \exp \left(\frac{i}{\hbar}\left(\epsilon_{\nu}-\epsilon_{\mu}\right) t\right) .
\end{array}
$$

This system is readily solved in terms of a collective model, e.g., the RVM. ${ }^{19}$ Taking $t=0$ to correspond to the distance of closest approach, the time-dependent interaction, $V$, has a duration of order $2 T$, so that $V(T) \approx 0$, after which time the solutions of Eq. (2.7) are constant, i.e., $a_{\nu}(t)$ $\approx a_{v}(T)$, for $t>T$. For such times the system of equations, Eqs. (2.6a), (2.6b) can be approximated by

$$
\begin{aligned}
i \hbar \dot{a}_{\nu}(t) \simeq & \int d E b_{E}(t)\left\langle\phi_{\nu}\left|H_{c}\right| \phi_{E}\right\rangle \\
& \times \exp \left(\frac{i}{\hbar}\left(\epsilon_{\nu}-E\right) t\right),
\end{aligned}
$$

$$
\begin{array}{r}
i \hbar \dot{b}_{E}(t) \simeq \sum_{\mu} a_{\mu}(t)\left\langle\phi_{E}\left|H_{c}\right| \phi_{\mu}\right\rangle \\
\times \exp \left(\frac{i}{\hbar}\left(E-\epsilon_{\mu}\right) t\right) .
\end{array}
$$

These equations describe the radioactive decay of the quasibound states due to their coupling to the continuum. They can be treated by standard Wigner-Weisskopf damping theory. The result is that the quasibound levels decay exponentially with a decay constant given by the "golden rule" formula

$$
\lambda_{\mu}=\left.\frac{2 \pi}{\hbar} \cdot\left\langle\phi_{E}\left|H_{c}\right| \phi_{\mu}\right\rangle\right|^{2} \text {. }
$$

The probability of fission of a given level is given by the standard formula involving the widths $\Gamma=\hbar \lambda$ for fission vs competing processes,

$$
p_{\mu}=\frac{\Gamma_{\mu}(\text { fission })}{\Gamma_{\mu}(\text { fission })+\Gamma_{\mu}(\text { gamma decay })+\cdots} .
$$

For the two-step process, Coulomb excitation followed by spontaneous fission, the fission cross section is given by

$$
\sigma_{\mathrm{CF}}=\sigma_{R} \sum_{\mu}\left|a_{\mu}(T)\right|^{2} p_{\mu} \equiv \sigma_{R} P^{\mathrm{CF}}
$$

in which $\sigma_{R}$ is the Rutherford scattering cross section. A simple alternative to Eq. (2.9) is to use barrier penetration theory. One finds, for states above the barrier top, using reasonable estimates of gamma and neutron widths, that $\Gamma_{\mu}($ fission $) \gg \Gamma_{\mu}($ gamma $)+\cdots$, so that $p_{\mu} \approx 1$. For states below the barrier, the penetrability falls rapidly with energy, resulting in completely negligible values of $p_{\mu}$ for states of the order of an $\mathrm{MeV}$ below the barrier top. As a result, in the present paper, we utilize the ansatz,

$$
\sigma_{\mathrm{CF}}=\sigma_{R} \sum_{\mu}\left|a_{\mu}(T)\right|^{2}
$$

in which the sum is over quasibound states above the barrier.

Three effects, which will be discussed in detail in a later paper, ${ }^{14}$ are neglected in this simple approach. These are (1) Eq. (2.12) underestimates $\sigma_{\mathrm{CF}}$ by neglecting contributions from states below the fission barrier. Of these neglected states; only those very close to the barrier top are important, 
and there is a relatively small number of these, compared to the number above the barrier. Therefore, we feel their neglect is a good approximation. (2) The damping due to the spreading of the collective state over nearly noncollective states has been neglected. For highly excited states of low spin, this damping results in a significant reduction in the fission probability. Such states are located in regions in which the density of noncollective states is high. The collective state is fragmented over many noncollective states which have relatively large $\gamma$ widths for $E 1$ and $M 1$ decay. The total fission probability of the states over which the collective state is fragmented, relative to other modes of decay, can be significantly less than that of the pure collective state. As will be shown in later sections, such low-spin states play a small role in the total fission cross section; therefore, the error introduced by overestimating their contributions in Eq. (2.12) is not serious. This error tends to compensate the underestimate (1). (3) The lifetimes of the most highly excited states considered in our calculations are of the order of the collision time; for these states, the validity of the two-step model is in question. This is under study.

The ansatz, Eq. (2.12), has the great virtue that it depends only on the height of the fission barrier. This is known experimentally for low spins. For higher spins, results are quoted for two very reasonable choices of the variation of moment of inertia with deformation. On the other hand, while Eq. (2.11) is more correct in principle, its application is highly model dependent.

\section{RESULTS}

A. The collective energy levels

The semiquantal Coulomb fission formalism described in the preceding section is quite general and does not depend on the precise structure of the collective nuclear Hamiltonian. $H_{c}$ has to be specified only for evaluating the spontaneous and stimulated matrix elements entering Eqs. (2.6). A dynamic two-center model (TCM) in which one may identify the distance vector $\vec{R}$ between both fragments with the fission degree of freedom would probably be the most complete and reliable way to attack the problem since it correctly reproduces the asymptotic behavior, i.e., necking in and separation into two fragments. It also would allow us to include nucleon transfer processes straightforwardly and thus to extend the present investigations to treat Coulomb and transfer fission as two competing and partially coherent processes as suggested in a recent article by Leigh et al. ${ }^{20}$ This would be of importance at energies close to the Coulomb barrier. The advantage of the TCM is to allow for a precise formulation of the various processes. The main problem associated with a TCM approach is, however, that for any practical calculation of the collective wave functions (e.g., for fixed deformation, neck, mass, and charge asymmetry parameters) and of the excitation process, strong refinements are necessary in order to prevent huge numerical computations.

A one-center description which we prefer in the following has the advantage that all degrees of freedom may be treated dynamically, and closed analytical expressions can be derived for the Coulomb and nuclear coupling potential with the projectile. The collective motion is specified by the surface variables $\alpha_{l_{m}}$ defined by the expansion of the nuclear surface into spherical harmonics

$$
R(\Omega)=R_{0}\left(1+\sum_{l, m} \alpha_{l m} Y_{l m}^{*}(\Omega)\right) .
$$

We restrict ourselves to the five coordinates $\alpha_{2 m}(m=-2, \ldots,+2)$ because quadrupole distortions are most strongly excited by the Coulomb interaction. The influence of higher multipoles will be discussed at the end of this section. Instead of dealing with the laboratory coordinates $\alpha_{2 m}$, it is advantageous to express the Hamiltonian in terms of the $\beta$ - and $\gamma$-vibrational parameters $a_{0}, a_{2}$ in the intrinsic main axis system and the Euler angles $\theta_{j}$.

In Sec. II we have shown that $P^{\mathrm{CF}}(t \rightarrow+\infty)$ can be related within reasonable approximations to the excitation amplitudes $a_{n}(+T)$ of the collective resonances in the vicinity of the fission barrier, Eq. (2.12). Hence, we do not need to evaluate the nonresonant continuum states $\phi_{E}$ explicitly. This considerably simplifies the nuclear structure calculations. In particular, we do not have to worry about the incorrect asymptotic behavior of the onecenter model because the resonance wave functions are concentrated at deformations $a_{0} \leqslant \beta_{s}$, where $\beta_{s}$ corresponds to the saddle point. In this work we take the bound states and approximate resonance positions of the actinide target nucleus from the collective rotation-vibration model (RVM) ${ }^{19}$ in which the complicated potential energy surface $V\left(a_{0}, a_{2}\right)$ is replaced by a two-dimensional harmonic oscillator. The RVM eigenfunctions exhibit the following structure:

$$
\begin{aligned}
&\left(H_{\mathrm{RVM}}-\epsilon_{\mu}^{J}\right) \phi_{\mu}^{J M}=0, \\
& \phi_{\mu}^{J M}\left(\theta_{j}, a_{0}, a_{2}\right)=\sum_{K, n_{2}, n_{0}} C_{\mu, K n_{2} n_{0}}^{J}\left|J M K\left(\theta_{j}\right)\right\rangle \\
& \times\left|K n_{2}\left(a_{2}\right)\right\rangle\left|n_{0}\left(a_{0}\right)\right\rangle,
\end{aligned}
$$

where $|J M K\rangle$ denotes the rotational part of the wave function and $\left|K n_{2}\right\rangle,\left|n_{0}\right\rangle$ account for the $\gamma$ and $\beta$ vibrations, respectively. The band mixing coef- 


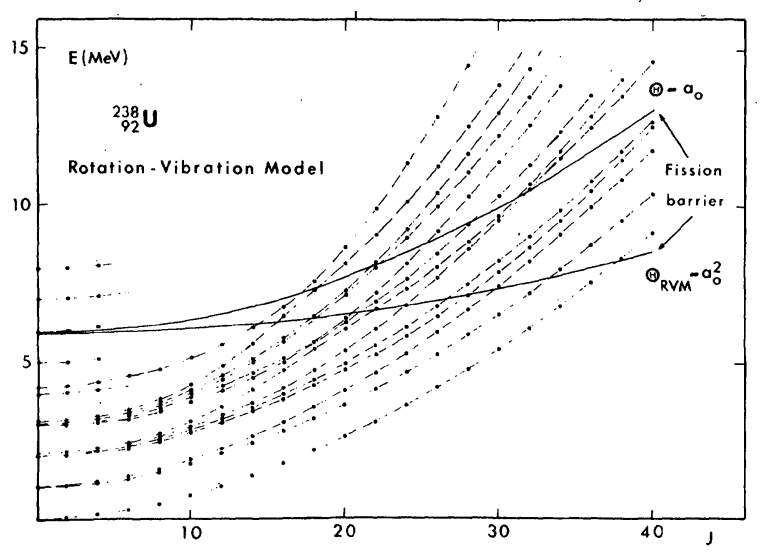

FIG. 1. Collective energy levels of ${ }^{238} U$ as predicted by the rotation-vibration model. The 13 lowest rotational bands built on top of the ground state and the $\beta$ - and $\gamma-$ vibrational states are indicated. In addition, the $J=0,2$, and 4 spin members of the $\beta$ vibrations up to 8 phonons are shown. The fission barrier as a function of spin has been evaluated for two different moments of inertia $\theta$.

ficients $C$ have to be calculated by a numerical diagonalization procedure.

Figure 1 displays the 13 lowest rotational bands built on top of the ground state and the various vibrational phonons. In order to study the influence of low-spin states on Coulomb fission, the $J=0,2$, and $4 \beta$ vibrations (up to 8 phonons) have also been taken into account. Rotation-vibration coupling due to the deformation dependence of the moments of inertia appreciably lowers the yrast band compared with the rigid rotor $J(J+1)$ level spacing. The RVM levels for the ground-state band agree quite nicely with experimental data obtained by Grosse et al. $^{21}$ at Berkeley and GSI. For a detailed comparison of the level spectrum and the $B(E 2)$ values, we refer to Ref. 22. Unfortunately, only a few low-spin vibrational states $(n=1)$ have been measured up to now; higher phonon states are completely unknown. The spin-dependent fission barrier

$$
E_{f}(J)=\max \left(V\left(a_{0}, a_{2}=0\right)+\frac{\hbar^{2} J(J+1)}{2 \Theta\left(a_{0}, a_{2}=0\right)}\right)
$$

has also been plotted in Fig. 1. $V$ is the empirical double-humped fission potential which has been deduced by Back et al. ${ }^{23}$ from $(t, p)$ reactions.

For finite spin, the inner barrier turns out to be higher than the outer one and therefore determines the fission cross section. The moment of inertia $\Theta$ at the first saddle point $\beta_{A}$ was calculated for two cases: (a) $\Theta_{\mathrm{RVM}} \sim a_{0}{ }^{2}$, and (b) $\Theta \sim a_{0}$. The former reproduces correctly the deformation dependence near the ground state (g.s.) minimum. At larger deformations $\theta \sim a_{0}$ seems to be favorable as may be seen from the experimental value for the rotational constant in the ${ }^{240} \mathrm{Pu}$ isomeric state. ${ }^{24,25}$ Such a behavior of the moment of inertia can be understood in the framework of the collective model by properly taking into account the higherorder corrections in the power series expansion of the collective kinetic energy ${ }^{19}$

$$
\begin{aligned}
T_{\text {coll }}\left(\pi_{2}, \alpha_{2}\right)= & \left(\sqrt{5} / 2 B_{2}\right)\left[\pi_{2} \otimes \pi_{2}\right]_{0}+B_{3}\left[\left[\pi_{2} \otimes \alpha_{2}\right]_{2} \otimes \pi_{2}\right]_{0}+B_{40}\left[\alpha_{2} \otimes \alpha_{2}\right]_{0}\left[\pi_{2} \otimes \pi_{2}\right]_{0} \\
& +B_{42}\left[\left[\alpha_{2} \otimes \alpha_{2}\right]_{2} \otimes\left[\pi_{2} \otimes \pi_{2}\right]_{2}\right]_{0}+B_{44}\left[\left[\alpha_{2} \otimes \alpha_{2}\right]_{4} \otimes\left[\pi_{2} \otimes \pi_{2}\right]_{4}\right]_{0}+\cdots
\end{aligned}
$$

The terms $\sim B_{3}, \sim B_{4 n}$ correspond to coordinate dependent mass parameters. Presently the RVM is extended by Seiwert ${ }^{6,26}$ to include the higher-order kinetic energy corrections. Assuming the same isomeric rotational constant for ${ }^{238} \mathrm{U}$ and ${ }^{240} \mathrm{Pu}$, we evaluated the moment of inertia at $\beta_{A}$ in case (b) by linear interpolation between $(\epsilon / 2)_{\text {g.s. }}$ and $(\epsilon / 2)_{2 \min }$ yielding $(\epsilon / 2)_{B_{A}}=4.3 \mathrm{keV}$. The Coulomb fission probabilities reported below are analyzed for both inertia parameters.

\section{B. Mean Coulomb excitation energy}

To get a feeling for the Coulomb excitation strength in very heavy-ion collisions, let us consider in Fig. 2 the mean excitation energy $\left\langle E^{*}\right\rangle$ of ${ }^{238} \mathrm{U}$ as a function of bombarding energy $E$ which here, and in the following, will be given in units of the Coulomb barrier ${ }^{10}$

$$
\begin{aligned}
& E_{\text {Coul }}=Z_{1} Z_{2} e^{2} / R_{\text {int }}, \\
& R_{\text {int }}=1.16\left(A_{1}^{1 / 3}+A_{2}^{1 / 3}+2\right)(\mathrm{fm}) .
\end{aligned}
$$

Different curves are drawn for ${ }_{54} \mathrm{Xe},{ }_{67} \mathrm{Ho},{ }_{82} \mathrm{~Pb}$, and ${ }_{92} \mathrm{U}$ projectiles. $\left\langle E^{*}\right\rangle$ was evaluated from the occupation amplitudes of the 256 states below and above the fission barrier (see Fig. 1) according to

$$
\left\langle E^{*}\right\rangle=\sum_{J, \mu} \epsilon_{\mu}^{J}\left|a_{\mu}^{J}(+T)\right|^{2}
$$

and therefore represents an average value for both Coulomb excitation and fission events. It is striking that the shapes are nearly independent of $Z_{p} ;$ only the absolute value increases with projectile charge number. Obviously, $E / E_{\text {Coul }}$ gives an appropriate scaling of the data.

For pure Coulomb interaction $\left\langle E^{*}\right\rangle$ increases monotonically with bombarding energy (dashed lines in Fig. 2). When strong interactions are 
taken into account, destructive Coulomb-nuclear (CN) interference becomes visible around $82 \%$ of the Coulomb barrier; the maximum excitation energy is deposited at $0.93 E_{\text {coul }}$. Details of the CN interference structure are certainly model dependent. We have employed a nuclear folding potential consisting of a Yukawa, compression, and symmetry term $^{22,27}$

$$
\begin{aligned}
V_{\text {coupl }}^{\text {nucl }}\left(\overrightarrow{\mathrm{r}}, \alpha_{2 m}\right)= & {\left[T_{l=2}^{\mathrm{Yuk}}(r)+T_{l=2}^{\text {comp }}(r)+T_{l=2}^{\text {sym }}(r)\right] } \\
& \times \sum_{m=-2}^{+2} \alpha_{2 m} Y_{2 m}^{*}(\Omega)
\end{aligned}
$$

The range $\mu$ and strength $V_{0}$ of the Yukawa transition potential

$$
\begin{aligned}
& T_{l=2}^{\mathrm{Yuk}}(\gamma)=V_{0} 8 \pi \rho_{1} \rho_{2} R_{01}{ }^{2} R_{02}{ }^{3} f_{2}(\gamma)\left[f_{2}\left(R_{02}\right)+f_{2}\left(-R_{02}\right)\right]\left[-f_{1}\left(R_{01}\right)+f_{1}\left(-R_{01}\right)\right], \\
& f_{1}(x)=\frac{1}{2} \exp (-x / \mu)\left[\left(\frac{\mu}{x}\right)+\left(\frac{\mu}{x}\right)^{2}\right], \quad f_{2}(x)=\frac{1}{2} \exp (-x / \mu)\left[\left(\frac{\mu}{x}\right)+3\left(\frac{\mu}{x}\right)^{2}+3\left(\frac{\mu}{x}\right)^{3}\right],
\end{aligned}
$$

were fixed to be $\mu=1.0 \mathrm{fm}$ and $V_{0}=-400 \mathrm{MeV} \mathrm{fm}$; this parameter choice ensures the occurrence of the interference minimum just at the Coulomb barrier, Eq. (3.5), but is, of course, not unique. For homogeneous density distributions $\rho_{1}$ and $\rho_{2}$, the compression and symmetry coupling potentials vanish in the nonoverlap region; they contribute only at incident energies exceeding the interaction barrier, $r \leqslant\left(R_{01}+R_{02}\right)$,

$$
\begin{gathered}
T_{l=2}^{\text {comp }}(r)=\frac{2 C}{\rho_{0}}\left(2 \rho_{1} \rho_{2}-\rho_{0}^{2}\right) R_{01}{ }^{2} R_{02}{ }^{3} g(r), \quad T_{l \neq 2}^{\text {sym }}(r)=\frac{G}{\rho_{0}}\left(1-2 \frac{Z_{1}}{A_{1}}\right)\left(1-2 \frac{Z_{2}}{A_{2}}\right) 4 \rho_{1} \rho_{2} R_{01}{ }^{2} R_{02}{ }^{3} g(r) \\
g(r)=4 \pi \sum_{m_{1}=0}^{2} \sum_{m_{2}=0}^{2} \sum_{m_{3}=0}^{1} \frac{\left(2+m_{1}\right) !}{m_{1} !\left(2-m_{1}\right) !} \frac{\left(2+m_{2}\right) !}{m_{2} !\left(2-m_{2}\right) !} \frac{\left(1+m_{3}\right) !}{m_{3} !\left(1-m_{3}\right) !}\left(-2 R_{02}\right)^{-m_{1}-1}(2 r)^{-m_{2}-1}\left(-2 R_{01}\right)^{-m_{3}-1} \\
\times \frac{\left(R_{02}+R_{01}-r\right)^{m+1}}{(m+1) !}
\end{gathered}
$$

where we have used the abbreviation $m=m_{1}+m_{2}$ $+m_{3} ; \rho_{0}=0.17 \mathrm{fm}^{-3}$ is the equilibrium density in nuclear matter. According to Scheid et al. ${ }^{19}$ the remaining constants are determined from binding energy calculations; the compression parameter $C$ is related to the nuclear compressibility $K$ by $C=K / 9 ;$ since $K \approx 300 \mathrm{MeV}$, we obtain $C \sim 30 \mathrm{MeV}$.

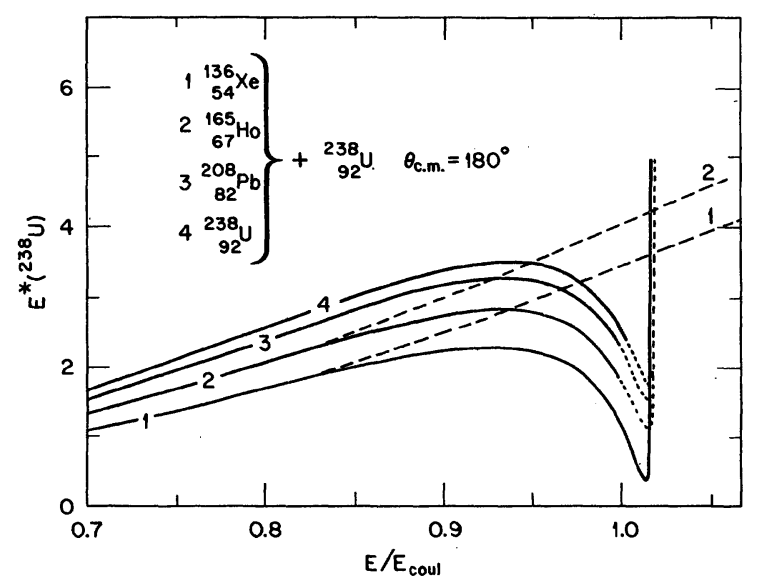

FIG. 2. Mean Coulomb excitation energy of ${ }^{238} \mathrm{U}$ for various projectiles and bombarding energies. The dashed curves have been calculated for a pure Coulomb potential, whereas the solid lines include destructive interference with the strong interaction (folded Yukawa potential).
The value of the symmetry constant $G$ can be fixed by requiring stability against $\beta$ decay, which yields $G \sim 70 \mathrm{MeV}$. The absolute $\left\langle E^{*}\right\rangle$ values are interesting, too; in all cases where the projectile energy is below $E_{\text {Coul }}$, even $U+U$, the mean excitation energy amounts to $3.5 \mathrm{MeV}$ at most, i.e., inelastic scattering is much more likely than fission. We would like to mention that interference structures in Coulomb excitation of rotational nuclei have recently been measured by Guidry et al. ${ }^{28}$ for ${ }^{40} \mathrm{Ar}$ and ${ }^{86} \mathrm{Kr}$ incident on ${ }^{232} \mathrm{Th}$ and ${ }^{238} \mathrm{U}$ targets. Since they show up also in Coulomb fission, it would be a formidable task to search for such a signature (see Figs. 3 and 4).

\section{Excitation functions for Coulomb fission at backward angles}

Figure 3 exhibits the Coulomb fission (CF) excitation function for $\mathrm{Xe}+\mathrm{U}$ in the c.m. frame at backward scattering angles. Qualitatively, the fission cross sections behave similarly to $\left\langle E^{*}\right\rangle$ : The solid lines have been calculated including destructive Coulomb-nuclear interference; the dotted, monotonically increasing curves belong to pure Coulomb interaction. In each case, the results are given for the two fission barriers shown in Fig. 1. Assuming the same moment of inertia in the second minimum of ${ }^{238} \mathrm{U}$ as measured by Specht et al. ${ }^{24}$ for ${ }^{240} \mathrm{Pu}$, the $\Theta \sim a_{0}$ result should be 


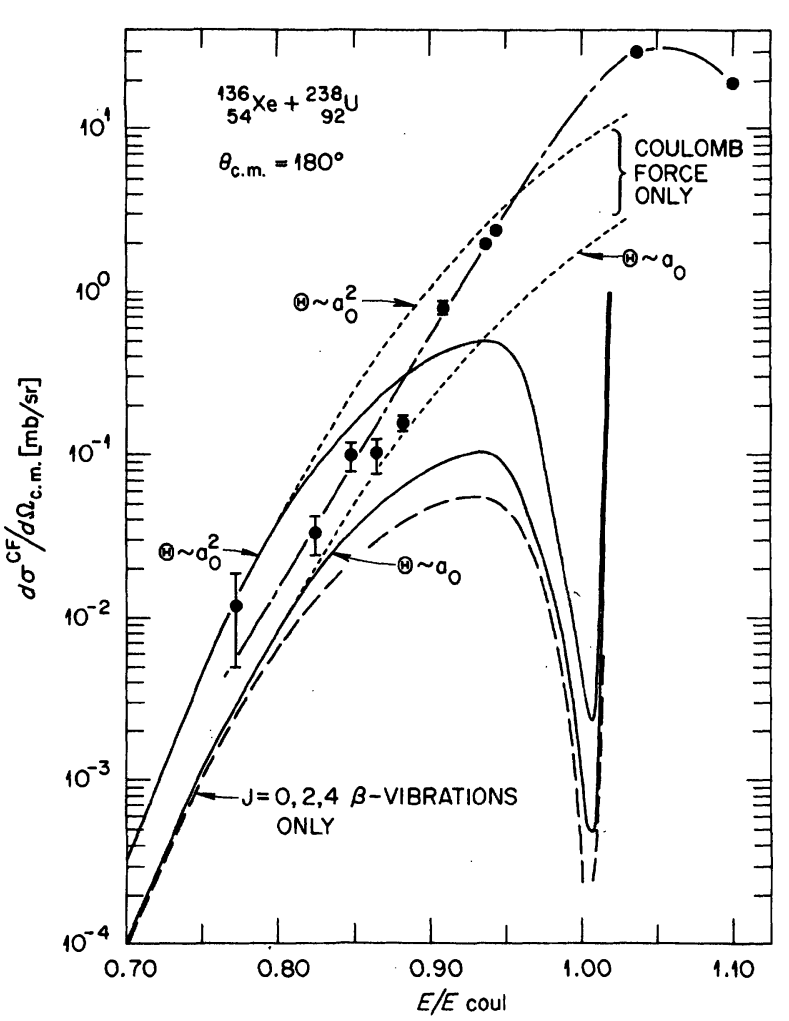

FIG. 3. Excitation function for Xe-induced Coulomb fission of ${ }^{238} \mathrm{U}$. The Coulomb barrier is defined in Eq. (3.5). Solid lines: present theory, including destructive Coulomb-nuclear interference, dotted curves: pure Coulomb coupling potential. Dashed line: contribution of the low-spin $\beta$ vibrations only. In each case, the results are given for the two spin-dependent fission barriers indicated in Fig. 1. The experimental data of Habs et al. (Refs. 11 and 29) represent an upper limit for Coulomb fission (see text); the dash-dotted line connecting the measured points is drawn to guide the eye. more realistic. In any case, the $\Theta \sim a_{0}$ and $\theta \sim a_{0}{ }^{2}$ curves depicted in Figs. 3, 4, and 9 allow for an easy interpolation once the rotational constant $(\epsilon / 2)_{2 \min }$ in ${ }^{238} U$ has been determined precisely. Near $E=0.93 E_{\text {Coul }}$ the differential cross section reachès its maximum value. Above the barrier the dominant Yukawa potential yields a steep rise of the fission probability. The dashed line gives the contribution of the low-spin $(J=0,2,4) \beta$ vibrations only. We will discuss it later. Recent experimental fission data for $\mathrm{Xe}+\mathrm{U}$ obtained by Habs et $a l .{ }^{29}$ at GSI are indicated, too. Within statistics, they agree with the results of similar counter experiments performed by Butler et al. ${ }^{10}$ at Berkeley which were restricted, however, to bombarding energies $E \geqslant 0.90 E_{\text {Coul }}$. We should stress that in both experiments the backscattered projectilelike nuclei have not been identified so that the measured points must be regarded an an upper limit for Coulomb fission. In a former GSI experiment ${ }^{11}$ the fission energy distribution has been determined additionally which helps to separate between different fission mechanisms. Habs et al. conclude that their data below $0.88 E_{\text {Coul }}$, where $\left\langle E_{f}\right\rangle$ amounts to $7.5 \pm 1.0 \mathrm{MeV}$, are compatible with dominant Coulomb fission whereas the events at higher bombarding energies must be attributed mainly to sequential fission following transfer or deep-inelastic reactions because of their much more negative $Q$ value. From the $Z_{p}$ independence of the cross section at $E>0.90 E_{\text {Coul }}$, Butler et al. are led to the same conclusion. Apparently, the GSI data below $88 \%$ of the interaction barrier are close to our theoretical cross section for $\Theta \sim a_{0}$, which indicates that CF is a dominant mechanism at low-incident energies. In the $\mathrm{CN}$ interference region the experimental sep-

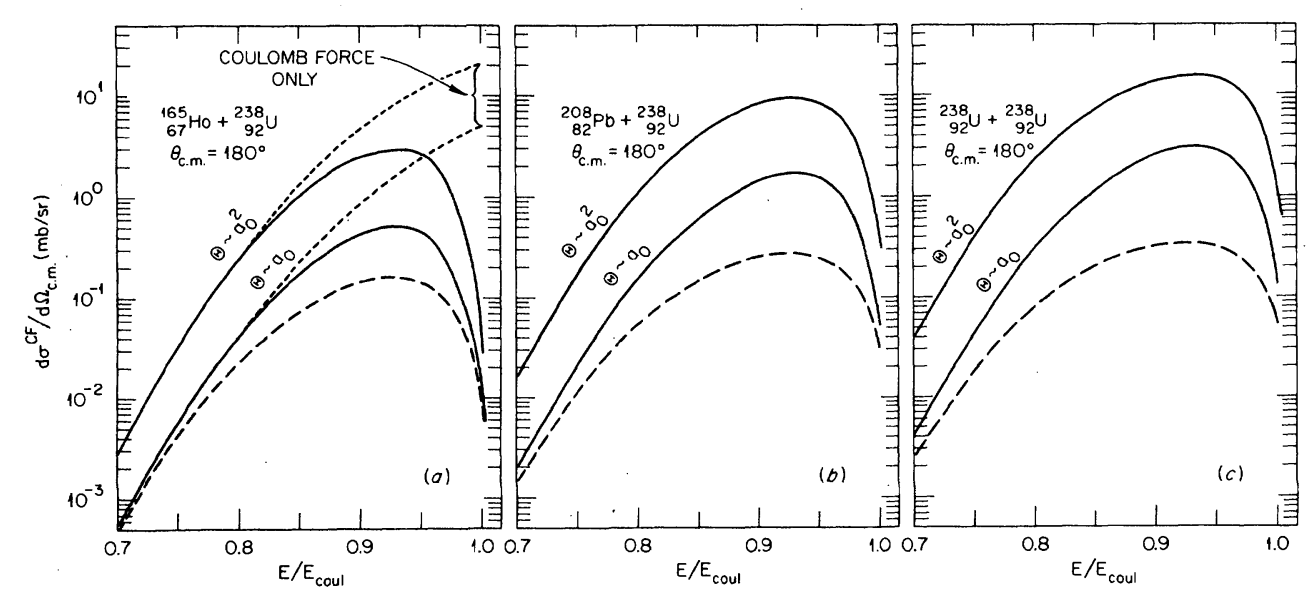

FIG. 4. Excitation functions at $\theta_{c_{0} m_{0}}=180^{\circ}$ for Coulomb fission of ${ }^{238} \mathrm{U}$ by different projectiles. The notation is the same as in Fig. 3. 
aration of Coulomb fission becomes much more laborious, since the dominating other fission channels have to be excluded by coincidence techniques.

Such an experiment will be performed at the super-HILAC in Berkeley utilizing a ${ }_{67}^{165} \mathrm{Ho}$ beam. ${ }^{30}$ Figure 4(a) shows our theoretical prediction for $\mathrm{Ho}+\mathrm{U}$. The shape of the excitation function plotted versus $E / E_{\text {Coul }}$ looks quite similar to the $\mathrm{Xe}+\mathrm{U}$ system, as is the case for $\mathrm{Pb}+\mathrm{U}$ and $\mathrm{U}+\mathrm{U}$, Figs. 4(b) and 4(c). However, the absolute value of the cross section increases strongly with $Z_{p \circ}$ This becomes even more transparent from Fig. 5, where the Coulomb fission probability of ${ }^{238} \mathrm{U}$ is depicted as a function of projectile charge $Z_{p}$ for several incident energies. In analogy to the shake-off process of the vacuum polarization cloud in superheavy electronic quasimolecules, ${ }^{31}$ Soff suggested a parametrization of the data in the form $P^{\mathrm{CF}}\left(180^{\circ}\right) \sim\left(Z_{p}\right)^{n}$ for a fixed ratio $E / E_{\mathrm{Coul}}$. Indeed we find such a scaling behavior in the region $50 \leqslant Z_{p} \leqslant 92$, where the exponent is $n \sim 6$. This

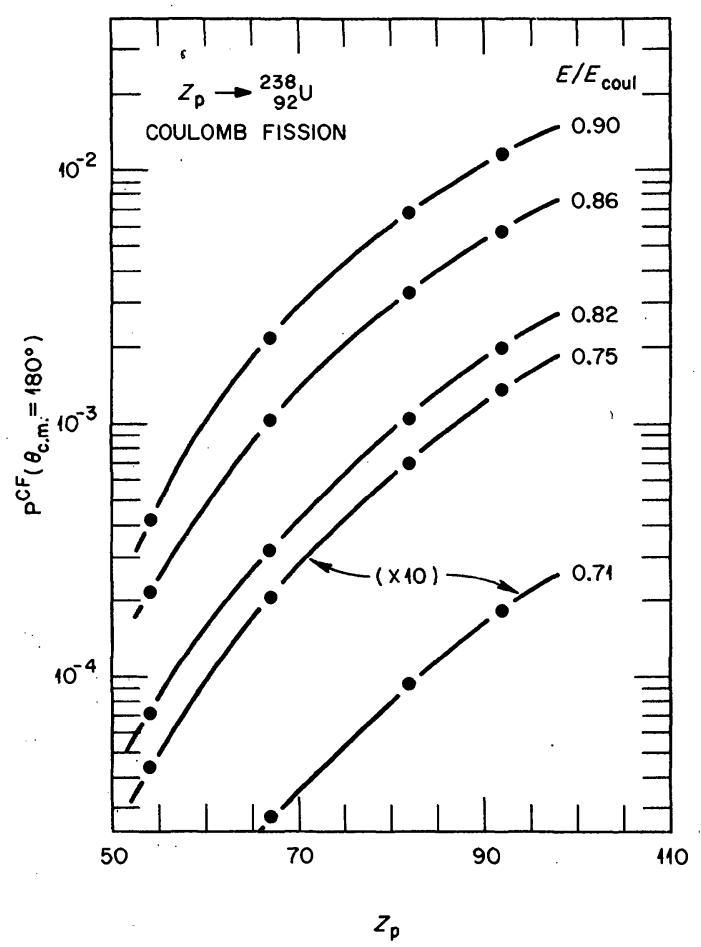

FIG. 5. Coulomb fission probability of ${ }^{238} \mathrm{U}$ as a function of projectile charge at backward scattering angles (c.m. frame). The results are depicted for several incident energies which are given in units of the Coulomb barrier, Eq. (3.5). For fixed ratio $E / E_{\text {Coul }}$, we find $P^{\mathrm{CF}} \sim\left(Z_{p}\right)^{6}$. The fission barrier $E_{f}(J)$ was evaluated with a moment of inertia $\Theta \sim a_{0}$. strong $Z_{p}$ dependence originates from the multiple excitation processes involved in Coulomb fission.

Let us discuss now the influence of collective rotations on CF. For this purpose, one has to compare the solid lines in Figs. 3, 4, and 9 (including all spin states) with the dashed lines which show the influence of the $J \leqslant 4 \beta$ vibrations only. For a moment of inertia $\Theta \sim a_{0}{ }^{2}$ the latter are nearly negligible. If we assume $\Theta \sim a_{0}$ to be more realistic, we find the interesting result that the importance of high-spin fission depends on bombarding energy, as well as projectile charge; the mean spin value $\langle J\rangle$ rises with increasing $E$ and $Z_{p}$. In the $\mathrm{Pb}+\mathrm{U}$ system, for example, the ratio $R$ between high-and low-spin contributions amounts to $R \sim 6$ at $E=0.93 E_{\text {Coul }}$, most of the cross section originates from the $J=16-20$ rotational members of the $n=3$ and 4 phonon states. Qualitatively, this behavior can be explained as follows: Fission from high-spin states requires 12 or more multiple $E 2$ Coulomb excitation steps (cf. Fig. 1) and becomes favorable if the combined coupling strength of Coulomb and nuclear interaction $V_{\text {coupl }}(t)$ is strong enough, i.e., for large $Z_{p}$ and small internuclear distance. If, however, the coupling remains relatively weak, for example, at low bombarding energies or in the CN interference region, few-step (and therefore low-spin) fission is preferred. In some earlier calculation $\mathrm{s}^{5 \cdot 6}$ where only the ground-state, $1 \beta$, and $1 \gamma$ band were taken into account and $\Theta_{\mathrm{RVM}} \sim a_{0}{ }^{2}$ was assumed, we have "forced" the nucleus to fission from the first $\beta$ band at spins $J \sim 28$ (see Fig. 1). If $\theta \sim a_{0}$ holds, this is no longer possible. The present theory avoids this shortcoming, since it does not make any a priori assumption about the spin distribution but allows the dynamics to decide what CF prefers.

\section{Fission fragment angular distributions}

Figure 6 shows the angular distribution of the fission fragments with respect to the beam axis in a central $\mathrm{Xe}+\mathrm{U}$ collision. It is most easily evaluated at $\theta_{\text {c.m. }}=180^{\circ}$ since the beam and recoil axes have the same direction, and only $M=0$ magnetic substates of the even-even target nucleus are populated. Our theoretical concept is based on two assumptions:

(a) The deformed actinide nucleus is assumed to separate along its symmetry axis. Since we describe the transition nucleus by the RVM wave functions $\phi_{\mu}^{J M}\left(a_{0}, a_{2}, \theta_{j}\right)$ in Eq. (3.2) this statement means that the fission angle $\theta_{f}$ coincides with the Euler angle $\theta_{2}$ between beam direction and symmetry axis. If just one of the eigenstates were excited, we would obtain 


$$
\begin{aligned}
\frac{d P_{\mu}^{J, M=0}\left(\theta_{2}\right)}{\sin \theta_{2} d \theta_{2}} & =\int_{0}^{2^{\pi}} d \theta_{1} \int_{0}^{2^{\pi}} d \theta_{3} \iint d a_{0} d a_{2}\left|\phi_{\mu}^{J, M=0}\left(a_{0}, a_{2}, \theta_{j}\right)\right|^{2} \\
& =\left(J+\frac{1}{2}\right) \sum_{K, m_{2}, m_{0}}\left|C_{\mu, K n_{2} n_{0}}^{J}\right|^{2}\left|d_{M=0, K}^{J}\left(\theta_{2}\right)\right|^{2},
\end{aligned}
$$

where use has been made of Eq. (3.2).

Secondly, we assume that the transition from the saddle point, where the resonances $\phi_{\mu}^{J M}$ are localized, to scission is so fast that the $K$ distribution (projection of $J$ onto the symmetry axis) is "frozen in." The validity of these assumptions has been well established by fission experiments. ${ }^{32}$

In reality not only one transition state will be excited by Coulomb coupling; we will have a rather broad level distribution. Then the above consideration has to be generalized and yields

$$
\frac{d P^{C F}\left(\theta_{2}\right)}{\sin \theta_{2} d \theta_{2}}=\sum_{J, \mu}\left|a_{\mu}^{J, M=0}(+T)\right|^{2}\left(J+\frac{1}{2}\right) \sum_{K, n_{2}, n_{0}}\left|C_{\mu, K_{n_{2} n_{0}}}^{J}\right|^{2}\left|d_{M=0, K}^{J}\left(\theta_{2}\right)\right|^{2},
$$

where $a_{\mu}^{J, M=0}$ are the excitation amplitudes. The sum over $\mu$ can be restricted to levels in the vicinity of the fission barrier. One might wonder why no interference terms between different levels

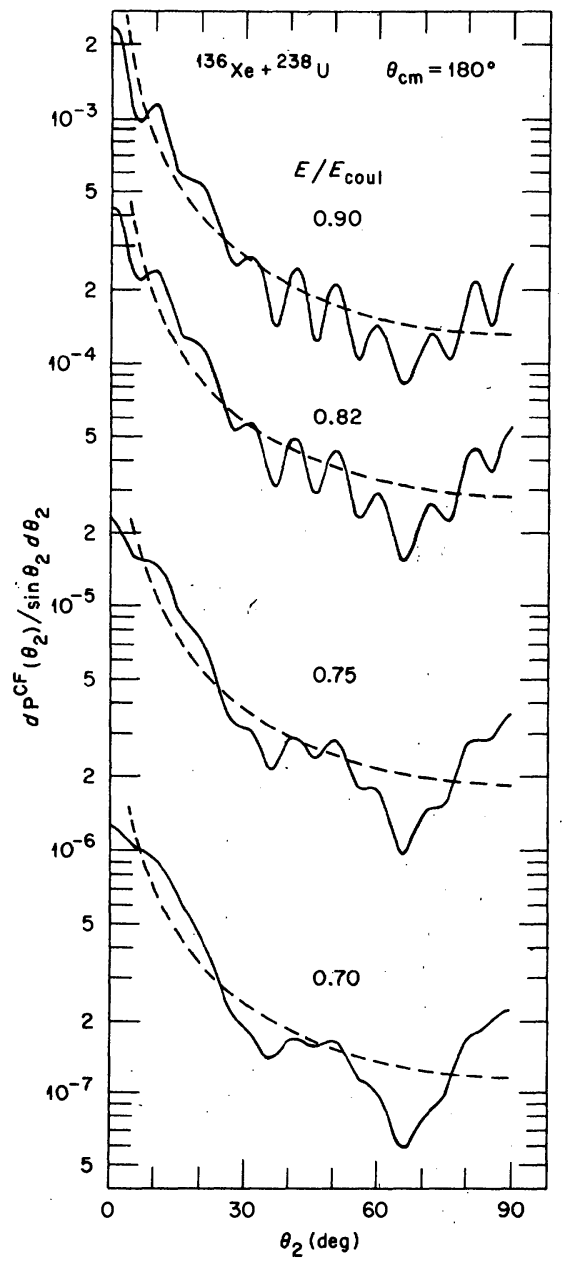

FIG. 6. Angular distribution of the fission fragments in the ${ }^{238} \mathrm{U}$ rest frame. The results correspond to a spin-dependent fission barrier with $\Theta \sim a_{0} . \mathrm{A} 1 / \sin \theta_{2}$ distribution (dashed line) is also shown for comparison.
$(J, \mu)$ appear on the right-hand side of Eq. (3.11). Indeed, it is characteristic for the semiquantal density matrix that it contains elements connecting different energy levels. This is because time is completely well defined in this picture and, consequently, the energy of the nucleus after the collision is undefined. A correct quantum mechanical treatment shows that one has to drop the nondiagonal terms proportional to $a_{\mu \prime}^{J^{\prime} M^{\prime}} a_{\mu}^{J_{M}}$ except for those belonging to different magnetic substates of the same level (see Ref. 15, Sec. III). These terms, however, do not occur in the special case of backward scattering since $M=M^{\prime}=0$.

From Fig. 6 we conclude that the fragment angular distribution in a head-on collision peaks at $\theta_{f}=0^{\circ}$ and $180^{\circ}$, i.e., in the beam direction and opposite to the beam which was already predicted by our earlier calculations where an approximate expression for the rotational matrices has been used. $^{5}$ For $\mathrm{Xe}+\mathrm{U}, d P^{\mathrm{CF}} / d \Omega_{f}$ approaches a $1 / \sin \theta_{f}$ distribution (dashed line) at incident energies $E$ $\sim 0.85 E_{\text {Coul }}$ which is in agreement with the presently available experimental data. ${ }^{10,11}$ On the other hand, the angular distribution for sequential fission following transfer reactions is also expected to be $\sim 1 / \sin \theta_{f}$, so that it seems difficult to identify Coulomb fission unambiguously by a $d P / d \Omega_{f}$ analysis. However, with decreasing projectile energy, systematic deviations occur which should be measurable. In Fig. 7 we have plotted the excitation probabilities of the collective resonances above $E_{b}=5.9 \mathrm{MeV}$ ver sus excitation energy $E^{*}$, i.e., the CF energy spectrum. The width of the distribution (several $\mathrm{MeV}$ ) and the mean value $\left\langle E_{f}\right\rangle$ increase with bombarding energy.

\section{E. Impact parameter dependence and} total cross sections

Let us now investigate the impact parameter dependence of Coulomb fission in the ${ }^{132} \mathrm{Xe}+{ }^{238} \mathrm{U}$. System (Fig. 8). At bombarding energies $E \leqslant 0.90 E_{\text {Coul }}$ the cross section $d \sigma^{\mathrm{CF}} / d \Omega_{\text {c.m. }}$ rises steeply with in- 


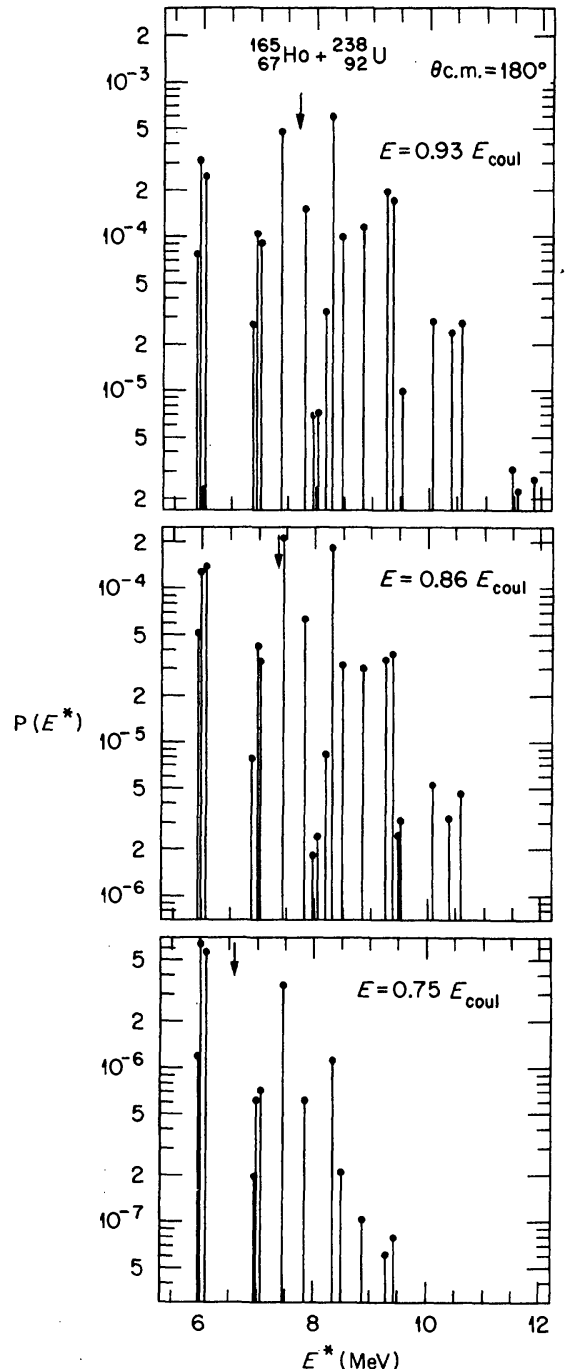

FIG. 7. Coulomb fission energy spectrum of ${ }^{238} \mathrm{U}$. The mean value $\left\langle E_{f}\right\rangle$ is marked by an arrow.

creasing scattering angle reaching its maximum value at backward scattering angles. In the numerical calculations only the $M=0$ magnetic substates of the levels shown in Fig. 1 were taken into account. This is exact at $\theta_{\text {c.m. }}=180^{\circ}$; without this limitation, the number of coupled differential equations would have become too large. This approximation is known to be justified at $\theta_{\text {c.m. }} \gtrless 120^{\circ}$ where most of the cross section originates and has been tested in connection with positron production by nuclear Coulomb excitation. ${ }^{33}$ We expect this simplification to underestimate the total cross sections by about $15 \%$. For completeness, we would like to note that the corresponding differential cross sections with respect to ion angle $\theta_{\text {c.m. }}$ (instead of solid ion angle $\Omega_{\text {c.m. }}$ ) show a peak near $\theta_{\text {c.m. }} \sim 150^{\circ}$ in the same energy domain.

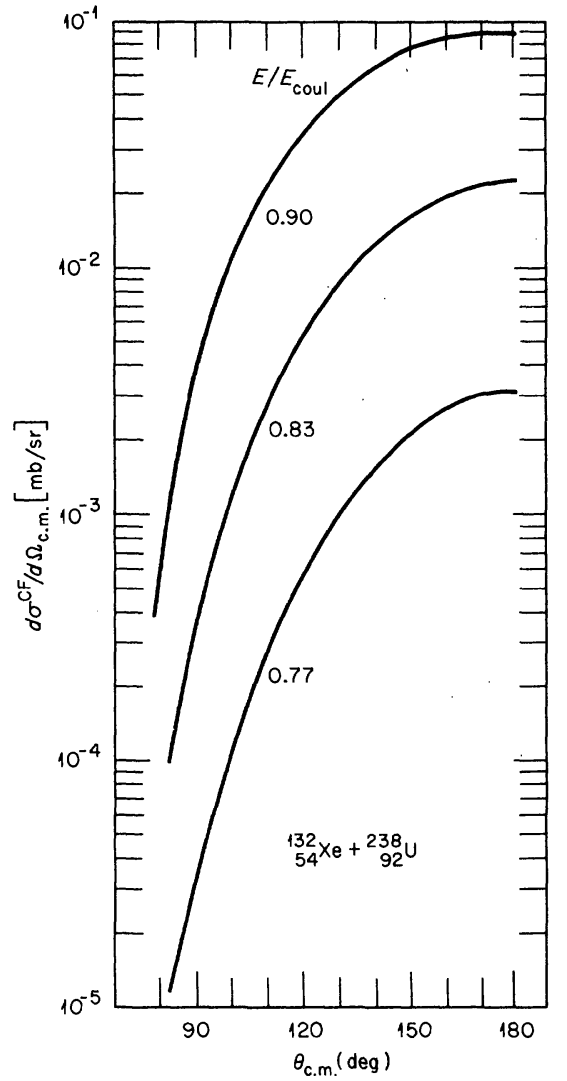

FIG. 8. Dependence of the differential $\mathrm{CF}$ cross sections on the scattering angle of the projectile; for a fission barrier $E_{f}(J)$ assuming $\Theta \sim a_{0}$.

By integration we obtain the total $\mathrm{CF}$ cross sections as functions of bombarding energy. Figure 9 gives a comparison between the theoretical results and recent radiochemical data measured at GSI. ${ }^{12}$ Fission events down to $E=0.71 E_{\text {Coul }}$ have been recorded corresponding to a distance of closest approach $R_{\min }=21.7 \mathrm{fm}$. In addition, cross sections for several stripping and pick-up reactions could be deduced. Although transfer reactions are observed even below $0.85 E_{\text {Coul }}$, there is strong evidence for a predominance of Coulomb fission in this energy region. If the ${ }^{238} \mathrm{U}$ target is bombarded with different $\mathrm{Xe}$ isotopes, the fission cross section remains the same within statistical errors, as is seen from Fig. 9. This behavior can hardly be explained by sequential fission following transfer, because transfer is known to be extremely sensitive to the $Q_{g g}$ values which change appreciably with mass number. Coulomb fission, on the other hand, depends only on the projectile charge, except for a slight change due to somewhat different kinematics that can easily be calculated. The energy dependence, as well as the order of magnitude, of the radiochemical fission data is rea- 


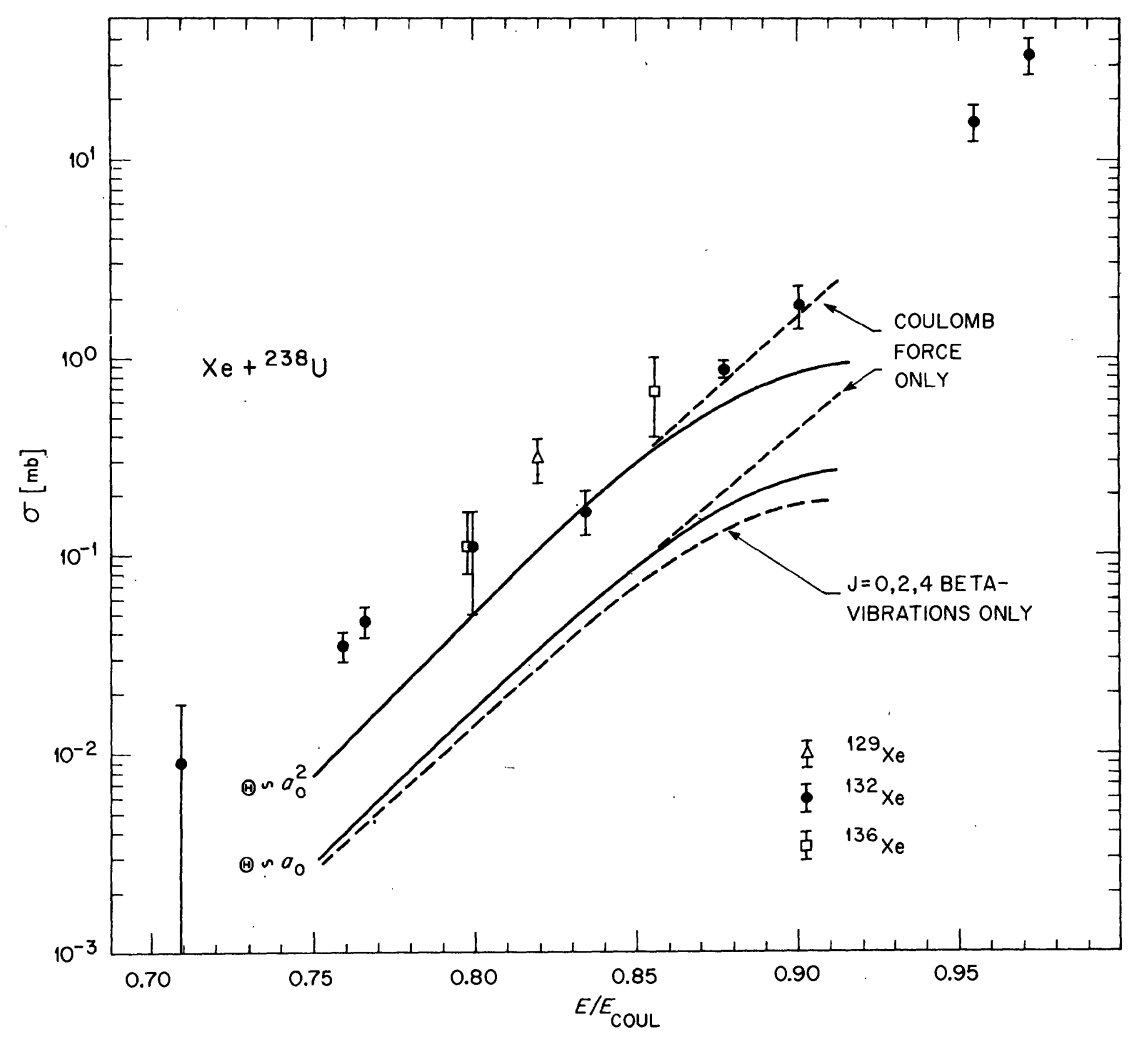

FIG. 9. Total Coulomb fission cross sections as a function of bombarding energy for ${ }^{132} \mathrm{Xe}+{ }^{238} \mathrm{U}$. The notation is the same as in Fig. 3. The radiochemical fission data (Ref. 12) taken at GSI represent an upper limit for Coulomb fission.

sonably well described by our theory. To save computing time, we have not evaluated any total cross sections at bombarding energies exceeding $0.90 E_{\text {Coul }}$. As in the case of backward scattering (Fig. 3), they are expected to exhibit a maximum near $0.93 E_{\text {Coul }}$ and reach the Coulomb-nuclear interference minimum just at the interaction barrier. Since a predominance of transfer-induced fission is very likely in this energy region, interference effects have not been observed either in the radiochemical or the counter experiments.

While preparing this paper, we have received a preprint by Levit and Smilansky. ${ }^{34}$ In contrast to our model which is essentially quantum mechanical, they develop a path integral formalism to describe the Coulomb fission process. The classically forbidden transitions are accounted for by complex trajectories. Their computations involve pure Coulomb coupling only, i.e., no Coulombnuclear interference, and are restricted to $\theta_{\text {c.m. }}$. $=180^{\circ}$. Only the $\beta$ vibrational degree of freedom is considered and rotations and $\gamma$ vibrations are neglected. According to our results, at least the neglection of rotation seems to be doubtful; this is the main reason why the fission fragment angular distribution in both theories looks quite different; Levit and Smilansky obtain a $\left|P_{2}\left(\cos \theta_{f}\right)\right|^{n}$ distribu- tion. The dependence on bombarding energy, however, is rather similar in both calculations of the $\mathrm{Xe}+\mathrm{U}$ system, but our cross sections are larger by a factor of 2 . In a forthcoming article ${ }^{14}$ we will give a detailed comparison between both theoretical approaches.

\section{F. Higher multipole vibrations and giant resonances}

Up to now, we have restricted the collective model space to quadrupole vibrations which are expected to give the dominant contribution to Coulomb fission. In contrast to several other theories $^{3 \times 34}$ all 5 degrees of freedom $\left(a_{0}, a_{2}, \theta_{j}\right)$ were treated dynamically. Higher multipole vibrations will certainly influence the fission cross sections. However, since the coupling potentials become much weaker with increasing multipolarity, it is unlikely that they might change the results significantly. There are, at present, uncertainties even in the quadrupole behavior of actinide nuclei. at excitation energies near $E_{f}$. Thus, a refinement of the theory in terms of octupole vibrations, which mainly account for the mass asymmetry in fission, and possibly hexadecapole shapes, does not appear to be useful at the moment. The in- 
fluence of these higher multipoles should be investigated after more sophisticated nuclear structure calculations based upon the complete quadrupole potential energy surface $V\left(a_{0}, a_{2}\right)$ have been finished. ${ }^{35}$ In this context, the coordinate-dependent mass parameters appearing in the collective kinetic energy, Eq. (3.4), have to be carefully studied. $^{26}$ They do not only influence the fission barrier $E_{f}(J)$, which was accounted for in this article, but may also modify the rotation-vibration interaction and hence the excitation amplitudes.

In the remainder of this section we would like to deal with giant resonances as doorways for Coulomb fission. Such resonances play an essential role in deep-inelastic reactions, ${ }^{36}$ but they are of minor importance in electromagnetic excitations at sub-Coulomb barrier energies. Because of their high energy $\Delta E$, they behave almost adiabatically. ${ }^{37}$ In the semiquantal formalism this follows from the large values of the adiabaticity parameter $(\xi \gg 1)^{15}$

$$
\xi\left(v, \theta_{\text {c.m. }}\right)=\frac{\Delta E}{\hbar} \frac{a}{2 v}\left[1+1 / \sin \left(\theta_{\text {c.m. }} / 2\right)\right],
$$

where the quantity $a$ denotes half the distance of closest approach at backward scattering and $v$ the velocity of the projectile (c.m. system). The $T=1$ giant dipole resonance is located at $E=11 \mathrm{MeV}$ $(\Gamma=3 \mathrm{MeV})$ in ${ }^{238} \mathrm{U}$, and the $T=0$ giant quadrupole resonance is concentrated at $E=10 \mathrm{MeV}(\Gamma=4$ $\mathrm{MeV})$. In the following we consider a $\mathrm{Xe}+\mathrm{U}$ collision with $E=0.85 E_{\text {Coul }}$ at $\theta_{\text {c.m. }}=180^{\circ}$ as an instructive example. The adiabaticity parameter for exciting the peak of the giant dipole resonance $(\Delta E$ $=11 \mathrm{MeV}$ ) turns out to be $\xi=5.160$ resulting in a negligible fission cross section. As will be discussed below, the dominant part of the cross section originates from the tail of the resonance at the fission barrier, i.e., $\Delta E=6 \mathrm{MeV}$. Even in this case, one obtains $\xi=2.814$.

For an order-of-magnitude estimate, it is justified to employ first-order perturbation theory,

$$
\begin{aligned}
\frac{d \sigma_{E L}\left(\theta_{\text {c.m. }}\right)}{d \Omega_{\text {c.m. }}}= & \left(\frac{Z_{1} \alpha}{v / c}\right)^{2} a^{-2 L+2} \frac{1}{e^{2}} B\left(E L, I_{0} \rightarrow I_{f}\right) \\
& \times \frac{d f_{E L}(\theta, \xi)}{d \Omega_{\text {c.m. }}},
\end{aligned}
$$

with $\alpha=1 / 137.03602$. The orbital integral functions $d f_{E L}$ have been extensively tabulated (see, e.g., Ref. 15). In applying Eq. (3.13), we have discretized the giant resonance continuum with a level spacing of $1 \mathrm{MeV}$ and evaluated the corresponding cross sections. The $B(E L)$ values for the discrete energy levels $E_{k}$ were obtained from the energy-weighted sum rule defined by

$$
\Sigma(E L)=\sum_{k}\left(E_{k}-E_{0}\right) \frac{1}{e^{2}} B\left(E L, E_{0}-E_{k}\right),
$$

yielding $^{38}$

$$
\Sigma(E 1)=\frac{9 \hbar^{2}}{8 \pi m} \frac{N Z}{A}
$$

and

$$
\Sigma(E L)=\frac{3 \hbar^{2}}{8 \pi m} Z L(2 L+1) R^{2 L-2} ; \quad L \geqslant 2,
$$

respectively; $m$ is the nucleon mass. In ${ }^{238} \mathrm{U}$, $100 \%$ of the sum rule is exhausted by the $T=1 \mathrm{di}-$ pole resonance and about $71 \%$ by the $T=0$ quadrupole resonance. ${ }^{39}$ For the reduced transition probabilities, a Lorentzian distribution was assumed.

At large adiabaticity parameters $(\xi>1)$ the functions $d f_{E L}$ in Eq. (3.13) decrease exponentially with $\xi$. Since $\xi$ is proportional to the excitation energy $\Delta E$, most of the fission cross section originates from the low-energy tail at $E_{f}=6 \mathrm{MeV}$. Hence, the $B(E L)$ strength concentrated at the fission barrier is the critical parameter determining $\sigma^{\mathrm{CF}}$.

Considering a $\mathrm{Xe}+\mathrm{U}$ collision at $E=0.85 E_{\text {Coul }}$, we find $d \sigma_{E_{1}}\left(180^{\circ}\right) / d \Omega_{\text {c.m. }}=3.0 \times 10^{-5} \mathrm{mb} / \mathrm{sr}$ and $d \sigma_{E_{2}}$ $\left(180^{\circ}\right) / d \Omega_{\text {c.m. }}=9.7 \times 10^{-5} \mathrm{mb} / \mathrm{sr}$ for the giant dipole and quadrupole resonance, respectively. These numbers have to be compared with a differential cross section of $3.6 \times 10^{-2} \mathrm{mb} / \mathrm{sr}$ (see Fig. $3, \theta$ $\sim a_{0}$ ) resulting for $\mathrm{CF}$ induced by multiple excitation of $\beta$-vibrational bands. Hence, we conclude that giant resonances as doorways for fission can be neglected in the projectile-target systems and energy regions which we studied.

However, in forward-scattering experiments with projectile energies well above the interaction barrier, giant resonance excitation becomes more favorable and has to be taken into account. This follows from the adiabaticity parameter, Eq. (3.12), yielding a ratio $\xi\left(\theta_{\text {c.m. }}=60^{\circ}\right) / \xi\left(180^{\circ}\right)=0.82$ for the $\mathrm{Xe}+\mathrm{U}$ system if the same minimal internuclear distance $r_{\min }\left(60^{\circ}\right)=r_{\min }\left(180^{\circ}\right)=18.2 \mathrm{fm}$ is chosen.

\section{SUMMARY AND CONCLUSIONS}

The results of the semiquantal theory outlined in Sec. II are in good agreement with recent counter and radiochemical experiments for the $\mathrm{Xe}+\mathrm{U}$ system. At sufficiently low bombarding energies $E$ $\leqslant 0.85 E_{\text {Coul }}$ where the experiments seem to indicate a predominance of Coulomb fission the shape, as well as the order of magnitude of the differential and total cross sections are well described.

The theory predicts several characteristic features of Coulomb fission which should be investigated in future experiments:

(a) There is a strong dependence of the CF probabilities on projectile charge number, $P^{\mathrm{CF}}\left(180^{\circ}\right)$ 
$\sim\left(Z_{p}\right)^{6}$ in the region $50 \leqslant Z_{p} \leqslant 92$ for fixed ratio $E / E_{\text {Coul }}$. This seems to be the most unique signature.

(b) The CF cross sections turn out to be very sensitive to the moment of inertia $\theta$ at the saddle point, in particular for projectiles with $Z_{p} \approx 70$, where spins up to $20 \hbar$ contribute. Hence, Coulomb fission might provide a tool to investigate $\theta$ at large deformations.

(c) There is a Coulomb-nuclear interference minimum similar to that found in inelastic scattering processes; its occurrence was already predicted several years ago by Holm and Greiner. ${ }^{4}$ Our present knowledge about transfer reactions suggests that such interference effects cannot be expected for sequential fission following particle transfer which is the strongest competing process.

(d) In those regions where Coulomb-nuclear interference can be neglected, the mean fission spin value $\left\langle J_{f}\right\rangle$ increases with $Z_{p}$ and bombarding ener gy.

(e) The fission fragment angular distribution exhibits deviations from $1 / \sin \theta_{f}$; the deviations are pronounced at low bombarding energies where low-spin states contribute most to the cross section.

In our opinion, the principal problems concerning the theory of Coulomb fission have been solved. Within a semiquantal method, we treat the continuum problem exactly by means of Feshbach's projection operator technique. The main uncertainties are expected to originate from our lack of knowledge of nuclear structure and from necessary approximations. To get even better agreement with experiments, additional investigations of the collective potential energy surface for actinide nuclei are necessary, in particular at high exci- tation energy. Both theoretical analyses ${ }^{26,35}$ and experimental nuclear structure studie ${ }^{40}$ are in progress; they mainly concentrate on ${ }^{238} \mathrm{U}$, but also the study of transuranic nuclei should be valuable.

We have estimated the influence of giant resonances as doorways for Coulomb fission. The results in first-order perturbation theory indicate that these are of minor importance for the projectile-target combinations and energy domains studied in this paper. However, at bombarding energies $E \gtrless 1.3 E_{\text {Coul }}$ and larger impact parameters (chosen such that transfer-induced fission can be neglected), the giant resonance contribution to $\mathrm{CF}$ will increase. For low $Z$ projectiles, e.g., ${ }_{36} \mathrm{Kr}$ incident on uranium, the relative influence of the giant resonances is expected to become larger, because these are excited in a onestep process, whereas multiple excitations of $\beta$ vibrations with large phonon numbers are strongly suppressed. In order to clarify the possible influence of giant resonances on Coulomb fission in high energetic collisions, more rigorous coupledchannels calculations should be performed in the future.

\section{ACKNOWLEDGMENT}

We are indebted to Dr. J. V. Kratz (GSI) and Professor Dr. H. J. Specht (Heidelberg) for their allowance to utilize some of their experimental data prior to publication. One of us (V.O.) acknowledges gratefully many stimulating discussions concerning Coulomb fission with members of the Physics Division staff of Oak Ridge National Laboratory. This research was sponsored by the Division of Physical Research, Department of Energy, under Contract No. W-7405-eng-26 with Union Carbide Corporation.
*Permanent address: Institut für Theoretische Physik, Johann Wolfgang Goethe Universität, 6000 Frankfurt am Main, West Germany.

${ }^{1}$ E. Guth and L. Wilets, Phys. Rev. Lett. 16, 30 (1966).

${ }^{2}$ L. Wilets, E. Guth, and J. S. Tenn, Phys. Rev. 156, 1349 (1967).

${ }^{3} \mathrm{~K}$. Beyer and A. Winther, Phys. Lett. 30B, 296 (1969); K. Beyer, A. Winther, and U. Smilansky, in Proceedings of the Intermational Conference on Nuclear Reactions Induced by Heavy Ions, Heidelberg, Germany, 1969 (North-Holland, Amsterdam, 1970), p. 804.

${ }^{4} \mathrm{H}$. Holm and W. Greiner, Phys. Rev. Lett. 26, 1647 (1971); Nucl. Phys. A195, 333 (1972).

${ }^{5} \mathrm{~V}$. Oberacker, G. Soff, and W. Greiner, J. Phys. G $\underline{3}$, L271 (1977).

${ }^{6} \mathrm{~V}$. Oberacker, G. Soff, and W. Greiner, in Nuclear Molecular Phenomena, edited by N. Cindro (North-Hol- land, Amsterdam, 1978); V. Oberacker, G. Soff, M. Seiwert, and W. Greiner, in Proceedings of the 16th International Winter Meeting on Nuclear Physics, Bormio, Italy, 1978, p. 220.

${ }^{7}$ C. E. Bemis, F. Plasil, R. L. Ferguson, E. E. Gross, and A. Zucker, Phys. Rev. C 10, 1590 (1974).

${ }^{8} \mathrm{C}$. Ngô, J. Péter, and B. Tamain, Nucl. Phys. A221, 37 (1974).

${ }^{9}$ P. Colombani, P. A. Butler, I. Y. Lee, D. Cline, R. M. Diamond, F. S. Stephens, and D. Ward, Phys. Lett. 65B, 39 (1976).

${ }^{10}$ P. A. Butler, I. Y. Lee, J. O. Newton, Y. El Masri, M. M. Aleonard, P. Colombani, R. M. Diamond, F. S. Stephens, R. W. Lougheed, and E. K. Hulet, Phys. Lett. 68B, 122 (1977).

${ }^{11}$ D. Habs, V. Metag, J. Schukraft, H. J. Specht, C. O. Wene, and K. D. Hildenbrand, Z. Phys. A283, 261 
(1977).

${ }^{12}$ G. Franz, J. V. Kratz, W. Brüchle, H. Folger, and B. Haefner, Z. Phys. A291, 167 (1979); J. V. Kratz (private communication).

${ }^{13}$ H. Kruse, W. T. Pinkston, W. Greiner, and V. Oberacker, J. Phys. G 5, L105 (1979).

${ }^{14} \mathrm{H}$. Kruse, W. T. Pinkston, V. Oberacker, and W. Greiner (to be published).

${ }^{15} \mathrm{~K}$. Alder and A. Winther, Electromagnetic ExcitationTheory of Coulomb Excitation with Heavy Ions (NorthHolland, Amsterdam, 1975).

${ }^{16}$ H. Feshbach, Rev. Mod. Phys. 36, 1076 (1964).

${ }^{17}$ W. L. Wang and C. M. Shakin, Phys. Lett. $32 \mathrm{~B}, 421$ (1970).

${ }^{18}$ M. Micklinghoff, Ph.D. thesis, University of Hamburg, West Germany, 1977 (unpublished).

${ }^{19} \mathrm{~J}$. M. Eisenberg and W. Greiner, Nuclear Theory' (North-Holland, Amsterdam, 1970), Vol. 1.

${ }^{20}$ J. R. Leigh, R. M. Diamond, A. Johnston, J. O. Newton, and S. H. Sie, Phys. Rev. Lett. 42, 153 (1979).

${ }^{21}$ E. Grosse, J. de Boer, R. M. Diamond, F. S. Stephens, and P. Tjøm, Phys: Rev. Lett. 35, 565 (1975); P. Fuchs, H. Bokemeyer, H. Emling, E. Grosse, D. Schwalm, H. J. Wollersheim, and D. Pelte, Jahresbericht 1976 GSI, Darmstadt, West Germany, GSI-J-177 (unpublished).

${ }^{22}$ V. Oberacker and G. Soff, Z. Naturf. 32a, 1465 (1977).

${ }^{23}$ B. B. Back, O. Hansen, H. C. Britt, and J. D. Garret, Phys. Rev. C 9, 1924 (1974).

${ }^{24}$ H. J. Specht, Rev. Mod. Phys. 46, 773 (1974).

${ }^{25}$ A. Sobiczewski, S. Bjørnholm, and K. Pomorski, Nucl. Phys. A202, 274 (1973).

${ }^{26} \mathrm{M}$. Seiwert, Diploma thesis, University of Frankfurt, West Germany, 1979 (unpublished).

${ }^{27}$ V. Oberacker, H. Holm, and W. Scheid, Phys. Rev. C 10,1917 (1974).

${ }^{28} \mathrm{M}$. W. Guidry et al., Phys. Rev. Lett. 40, 1016 (1978).

${ }^{29}$ D. Habs, V. Metag, D. von Harrach, P. Plässel, H. J. Specht, and J. Schukraft, MPI, Heidelberg, West Germany, Jahresbericht, 1978 (unpublished).

${ }^{30}$ C. F. Maguire, J. H. Hamilton, A. V. Ramayya, G. B. Bomar, and W. G. Nettles, Dept. of Physics, Vanderbilt University, Nashville, Tennessee, private communication.

${ }^{31} \mathrm{G}$. Soff, J. Reinhardt, B. Müller, and W. Greiner, Phys. Rev. Lett. 38, 592 (1977).

${ }^{32} \mathrm{R}$. Vandenbosch and J. R. Huizenga, Nuclear Fission (Academic, New York, 1973).

${ }^{33}$ V. Oberacker, G. Soff, and W. Greiner, Phys. Rev. Lett. 36, 1024 (1976).

${ }^{34}$ S. Levit and U. Smilansky, Nucl. Phys. A315, 205 (1979) .

${ }^{35}$ P. O. Hess, Diploma thesis, University of Frankfurt, West Germany, 1978 (unpublished); see also P. O. Hess, J. Phys. G 4 , L59 (1978).

${ }^{36}$ R. A. Broglia, C. H. Dasso, G. Pollarolo, and A. Winther, Phys. Rev. Lett. 41, 25 (1978).

${ }^{37}$ H. Holm, D. Scharnweber, W. Scheid, and W. Greiner, Z. Phys. 231, 450 (1970); H. Holm and W. Greiner, Phys. Rev. Lett. 24, 404 (1970).

${ }^{38} \mathrm{G}$. R. Satchler, Elementary Modes of Excitation in Nuclei (Soc. Italiana di Fisica, Bologna, Italy, 1977), pp. 271-351.

${ }^{39}$ J. A. Neto, S. B. Herdade, B. S. Bhandari, and I. C. Nascimento, Phys. Rev. C 18, 863 (1978).

${ }^{40}$ Th. W. Elze, E. Grosse, D. Schwalm, and H. J. Wollersheim, GSI, Darmstadt, private communication. 